\title{
Mode multiplexing at 2×20Gbps over 19-cell hollow-core photonic band gap fibre
}

\author{
Carpenter, Joel; Xu, Jing; Peucheret, Christophe; Wilkinson, Timothy D.
}

Published in:

OFC/NFOEC Technical Digest

Publication date:

2012

Document Version

Publisher's PDF, also known as Version of record

Link back to DTU Orbit

Citation $(A P A)$ :

Carpenter, J., Xu, J., Peucheret, C., \& Wilkinson, T. D. (2012). Mode multiplexing at 2×20Gbps over 19-cell hollow-core photonic band gap fibre. In OFC/NFOEC Technical Digest (pp. JW2A.41). Optical Society of America (OSA).

\section{General rights}

Copyright and moral rights for the publications made accessible in the public portal are retained by the authors and/or other copyright owners and it is a condition of accessing publications that users recognise and abide by the legal requirements associated with these rights.

- Users may download and print one copy of any publication from the public portal for the purpose of private study or research.

- You may not further distribute the material or use it for any profit-making activity or commercial gain

- You may freely distribute the URL identifying the publication in the public portal 


\title{
Mode Multiplexing at 2×20Gbps over 19-cell Hollow-Core Photonic Band Gap Fibre
}

\author{
Joel Carpenter ${ }^{1}$, Jing $\mathrm{Xu}^{2}$, Christophe Peucheret ${ }^{2}$, Timothy D. Wilkinson ${ }^{1}$ \\ 1 - Electrical Engineering Division, Engineering Department, University of Cambridge, 9 JJ Thomson Avenue, Cambridge, CB3 OFA, UK \\ 2 - DTU Fotonik, Department of Photonics Engineering, Technical University of Denmark, DK-2800 Kgs. Lyngby, Denmark. \\ jac240@cam.ac.uk
}

\begin{abstract}
This paper demonstrates the first mode-multiplexed system over 19-cell hollow-core photonic band gap fibre, at $2 \times 20 \mathrm{Gbps}$ using the $\mathrm{LP}_{0,1}$ and $\mathrm{LP}_{2,1}$-like modes.

OCIS codes: 060.0060 (Fiber optics and optical communications), 060.5295 (Photonic crystal fibers)
\end{abstract}

\section{Introduction}

Mode division multiplexing (MDM) has been recognized as a promising candidate for extending the capacity limit of single mode fibres (SMFs) [1-3] by utilizing the spatial dimension of optical fibres. MDM has been demonstrated previously using Graded-Index Multi-Mode Fibres (GI-MMFs) for short-range high-speed applications [4-5]. MDM targeting long-haul communication has been demonstrated so far in some Few-Mode Fibre (FMF) prototypes [1-3], which are all solid-core fibres. One of the well-known practical limitations of solid core fibres is fibre nonlinearities. Hollow-Core Photonic BandGap Fibres (HC-PBGFs) can greatly reduce nonlinearities as in excess of $90 \%$ of the power propagates through air [6]. For the same reason, these fibres also have the potential to offer decreased loss over solid fibres [7]. Increasing the dimension of the air core reduces loss but also results in an increase in the number of supporting modes [6]. This would be seen as undesirable if not for the potential of MDM to exploit this larger modal diversity. In [8] we explored MDM in a 7-cell HC-PBGF that only supports $\mathbf{L P}_{0,1}$ and $\mathbf{L P}_{1,1}$ modes. In this work, the air core of the HC-PBGF used for the mode transportation increases to 19 cells which supports approximately 20 degenerate modes per polarisation [6]. In addition, HC-PBGFs can be used for opening new transmission windows around $2 \mu \mathrm{m}$ where they have been theoretically predicted to have minimum loss [9] that is significantly lower than that of solid-core silica fibres in the same wavelength range.

In this work, a $2 \times 20 \mathrm{Gbps}$ two-mode system, employing the $\mathrm{LP}_{0,1}$ and $\mathrm{LP}_{2,1}$ modes, is demonstrated over a section of 19-cell HC-PBGF. Accurate mode excitation is enabled by a binary phase Spatial Light Modulator (SLM) and more than $10 \mathrm{~dB}$ crosstalk suppression is achieved by a standard $50 / 5050 \mu \mathrm{m}$ graded-index core multimode coupler. The experimental results reveal the possibilities, as well as challenges, of MDM in many-mode HC-PBGF.

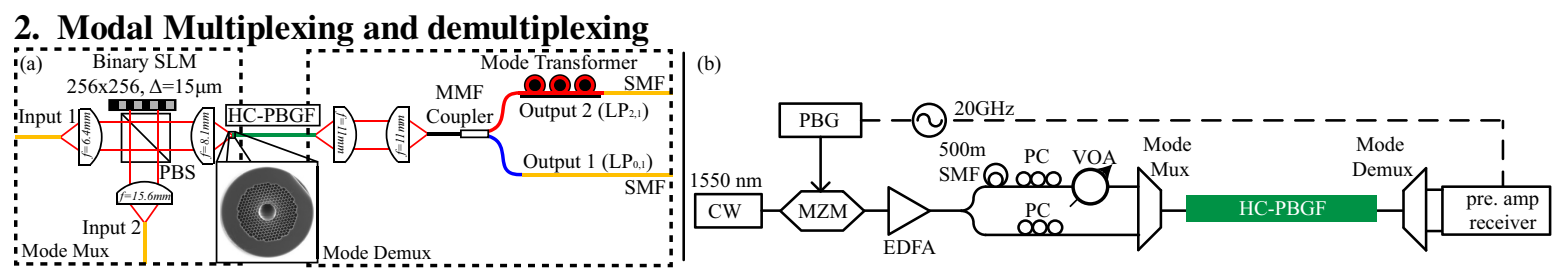

Fig. 1. (a) Modal multiplexing/demultiplexing mechanism. (b) System overview.

The system multiplexes channels on two separate modes of the fibre. The first channel propagates using the $\mathrm{LP}_{0,1}$ mode and is launched simply by resizing the fundamental mode of the input Single-Mode Fibre (SMF) as illustrated in Fig. 1(a). The second modal channel is launched by way of a binary phase SLM which displays a phase mask designed to generate the desired mode in the far-field [10]. The SLM can be programmed to excite any mode of the fibre at any orientation. The modes travel through a relatively short distance of fibre of $75 \mathrm{~cm}$. The limited distance is a consequence of the high Differential Mode Attenuation (DMA) of the fibre which can be several $\mathrm{dB} / \mathrm{m}$ [11]. For instance, at equal launch power there was a measured difference of $2.7 \mathrm{~dB} / \mathrm{m}$ between the $\mathrm{LP}_{0,1}$ and $\mathrm{LP}_{2,1}$ modes used for this experiment. At the receiving end, the similarity between the modes of the HC-PBGF and that of a standard multimode fibre is exploited by using a 50 $\mathrm{m}$ GI-MMF coupler, nominally rated as a 50/50 split at 850/1310nm, as the demultiplexer. Using a multimode coupler as a modal demultiplexer is a common method [5] of achieving the required modal diversity but is used in a slightly unusual fashion in this instance. First, rather than physically buttcoupling the two fibre cores together, the HC-PBGF core is imaged onto the coupler's core. The primary reason for employing this technique is to avoid physically touching the core of the HC-PBGF and so the two cores can be precisely aligned. This also allows a mirror to be inserted between the lenses to observe the far-field exiting the HCPBGF and being imaged onto the coupler at any given moment without moving the fibre and hence changing the 
mode profile. The MMF coupler itself as shown in Fig. 1(a) has two output ports labeled 1 (blue) and 2 (red) respectively. Any mode can couple to the first port but only the $\mathrm{LP}_{0,1}$ can couple through the SMF attached to it. The $\mathrm{LP}_{0,1}$ mode however cannot strongly couple to the second port which only transmits the higher-order modes. Because the desired system requires single-mode outputs, the second output port of the coupler is threaded through a polarisation controller which is used as a crude mode converter to maximize the coupling into the output singlemode fibre.

It is primarily the modal characteristics of the MMF coupler and the requirement for single-mode outputs which defines which mode is best for multiplexing the second channel. There are two contradictory requirements; the second channel must be sufficiently different from the fundamental so it is unlikely to couple to the SMF attached to the first output, but sufficiently similar to the fundamental that the simple mode transformer on the second port can be manipulated to provide adequate power to the SMF attached to that port and preserve the modal diversity between the two channels. Without the SMF attached to the second port, the isolation of the $\mathrm{LP}_{1,1}$ mode is between 10.5 and $12.5 \mathrm{~dB}$ depending on the orientation whereas the $\mathrm{LP}_{2,1}$ mode provides between 15.4 to $18 \mathrm{~dB}$. Higher-order modes provide similar isolation to that of the $\mathrm{LP}_{2,1}$ but suffer more loss, both through the HC-PBGF itself but more importantly, when an SMF is attached to the second port. The $\mathrm{LP}_{2,1}$ mode could be coupled to the SMF with $6 \mathrm{~dB}$ loss leaving $11-12 \mathrm{~dB}$ of isolation between the ports for that mode. Although the use of a SMF on the second port is an impediment to the isolation for the higher-order modes any additional loss through the second port serves to better isolate the $\mathrm{LP}_{0,1}$ channel. The leakage of the fundamental to the second port was measured to be around $21 \mathrm{~dB}$.

The Differential Mode Delay (DMD) of the fibre is very large, with just $75 \mathrm{~cm}$ of HC-PBGF having similar DMD to hundreds of metres or a few kilometres of graded-index multimode fibre and possesses even larger delays than step-index fibres designed specifically with large DMD for mode-multiplexing [12].The large difference in propagation constants of the modes serves to reduce the beat length and keep the propagating modes isolated. However the single-mode fibre on the second port preferentially couples lower order modes and tends to skew the final modal power distribution which can effectively amplify the modal dispersion for the secondary port. For example, if a channel is propagated using the $\mathrm{LP}_{2,1}$ mode, but some small mode-coupling occurs to the $\mathrm{LP}_{1,1}$ mode either at the launch or along the length of the fibre, the fact that the $\mathrm{LP}_{1,1}$ component of the power is more likely to couple to the single-mode output than is the $\mathrm{LP}_{2,1}$ may serve to boost the relative power of the $\mathrm{LP}_{1,1}$ mode and hence increase the modal dispersion. This also leads to an effect whereby adjusting the mode transformer to maximize the power out of the secondary port, also tends to make the modal dispersion worse, and in a similar fashion, rotating the orientation of the launched mode at the input of the fibre to maximise the power also tends to degrade isolation.
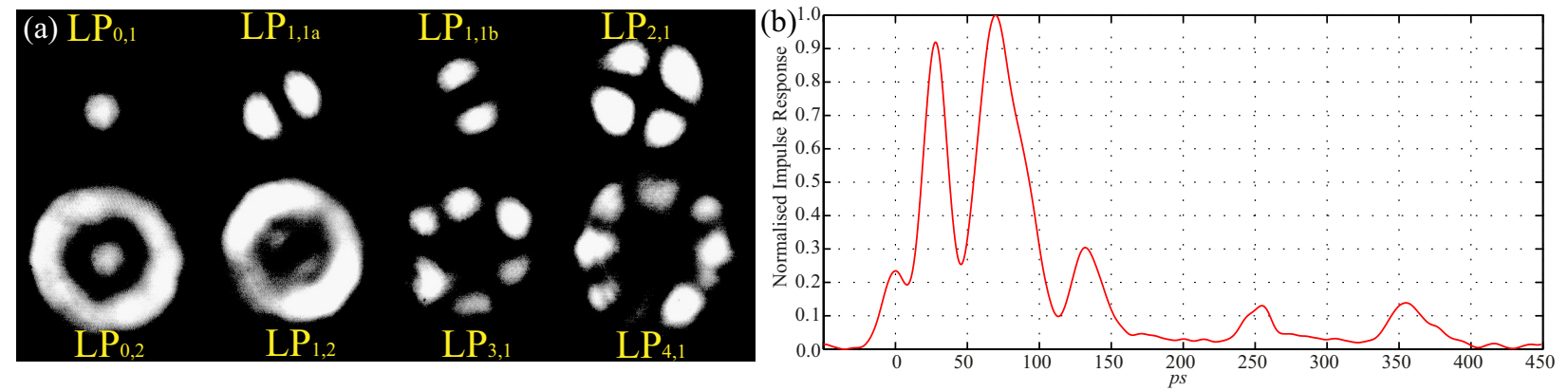

Fig. 2. (a) Far-field of the HC-PBGF at the receiving end (b) Impulse response after $75 \mathrm{~cm}$ with multiple modes excited simultaneously.

\section{Experimental setup and BER performance}

The experimental setup is shown in Fig. 1 (b). A continuous-wave (CW) light at $1550 \mathrm{~nm}$ is modulated by a MachZehnder modulator (MZM) and a 20 Gbps Non-Return to Zero (NRZ) signal with $2^{31}-1$ pseudo-random sequence length is generated. Two data channels which are to be propagated by the $\mathrm{LP}_{0,1}$ and $\mathrm{LP}_{2,1}$ modes respectively, are formed by splitting the signal at the output of the EDFA into two branches and decorrelating the channels by passing the $\mathrm{LP}_{0,1}$ path through a $500 \mathrm{~m}$ length of standard SMF. Polarisation controllers (PCs) are included in each path to align them to the polarisation required by the mode multiplexer. An attenuator is used in the $\mathrm{LP}_{0,1}$ channel path to balance the power of the two channels to keep them approximately equal at the receiver. The demultiplexed signal is sent into a pre-amplified receiver for bit error ratio (BER) measurements.

Fig. 3 shows the BER performance of the mode multiplexing system. The back-to-back case is obtained by sending the signal at the output of the MZM into the pre-amplified receiver directly. The eye diagram shown furthest to the left is measured in this case at $-25 \mathrm{dBm}$ received power. Compared to the back-to-back case, error-free single channel performances was achieved at $\mathrm{BER}=10^{-9}$ with a power penalty of 0.8 and $3.6 \mathrm{~dB}$ for $\mathrm{LP}_{0,1}$ (up triangle) and $\mathrm{LP}_{2,1}$ channel (down triangle), respectively. The $2.6 \mathrm{~dB}$ power penalty difference between $\mathrm{LP}_{0,1}$ and $\mathrm{LP}_{2,1}$ channels is 
due to modal dispersion as mentioned above. The modal performance of the demultiplexer is such that lower order modes can more easily couple to the single-mode outputs. This is not an issue for the modal bandwidth of the $\mathrm{LP}_{0,1}$ channel, but decreases the bandwidth of the $\mathrm{LP}_{2,1}$ as it effectively amplifies any modal mixing that has occurred to lower-order modes, due to the difference in modal attenuation when coupling from the MMF to the SMF.

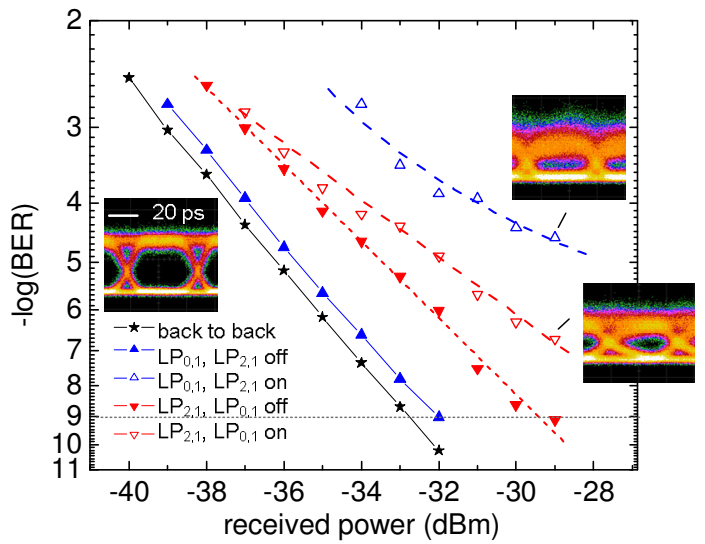

Fig. 3 BER performance. All eye diagrams are taken for a period of 30 seconds.

The maximum received power obtained in the experiment is $-28.3 \mathrm{dBm}$, which is set by the maximum power rating of the SLM. The crosstalk measured for the $\mathrm{LP}_{0,1}$ and $\mathrm{LP}_{2,1}$ channel is 21.2 and $11.3 \mathrm{~dB}$, respectively. With the both channels transmitting, BER down to $10^{-7}$ and $3 \times 10^{-5}$ has been confirmed for the $\mathrm{LP}_{2,1}$ and $\mathrm{LP}_{0,1}$ channels respectively at $-29 \mathrm{dBm}$ received power. The slope of the $\mathrm{BER}$ curve for $\mathrm{LP}_{0,1}$ and $\mathrm{LP}_{2,1}$ degrades heavily compared to the back-to-back case and indicates an error floor at higher received power levels for the $\mathrm{LP}_{0,1}$ channel. Whilst the $\mathrm{LP}_{2,1}$ channel's primary limitation is the exaggeration of modal dispersion by the demultiplexer, the $\mathbf{L P}_{0,1}$ channel suffers from increased leakage of the $\mathrm{LP}_{2,1}$, again due to the demultiplexing scheme where the increased loss introduced by attempting to couple from a multimode fibre to a single-mode output reduces the isolation between the channels.

\section{Conclusions}

Multiplexing and demultiplexing of two spatial modes of an HC-PBGF has been demonstrated with $2 \times 20 \mathrm{Gbps}$ NRZ channels. BER of $3 \times 10^{-5}$ is achieved for the worst case. Although the multiplexing of modes by the SLM was very effective, demultiplexing by way of a standard multimode coupler to two single-mode fibre outputs was the primary limitation as it degrades the isolation between the channels as well as exaggerates modal dispersion.

The authors would like to thank NKT photonics and especially Jens Kristian Lyngsø and Lasse Leick for providing the HC-PBGF. The authors would also like to thank Radu Malureanu for providing the infrared camera. This work is partly supported by the Danish Council for Technology and Productions Sciences (project 10-093299).

\section{References}

[1] Clemens Koebele, Massimiliano Salsi, Laurent Milord, Roland Ryf, Cristian Bolle, Pierre Sillard, Sébastien Bigo, Gabriel Charlet, "40km Transmission of Five Mode Division Multiplexed Data Streams at 100Gb/s with low MIMO-DSP Complexity," in ECOC 2011, paper Th.13.C.3. [2] A. Li, A. Al Amin, X. Chen and W. Shieh, "Reception of Mode and Polarization Multiplexed 107-Gb/s CO-OFDM Signal over a Two-Mode Fiber," in OFC 2011, paper PDPB8.

[3] R. Ryf, S. Randel, A. H. Gnauck, C. Bolle, R.-J Essiambre, P.J. Winzer, "Space-division multiplexing over 10km of three-mode fibre using coherent 6x6 MIMO processing," in OFC 2011, paper PDPB10.

[4] S. Schöllmann, S. Soneff, W. Rosenkranz, "10.7Gb/s Over 300 m GI-MMF Using a 2x2 MIMO System Based on Mode Group Diversity Multiplexing," in OFC 2007, paper OTuL2.

[5] B. C. Thomsen, "MIMO enabled 40Gb/s transmission using mode division multiplexing in multimode fiber," in OFC 2010 , paper OThM6.

[6] M. N. Petrovich, et. al, "Robustly single mode hollow core photonic bandgap fiber," Optics Express 16, pp. 4337-4346 (2008).

[7] P. Roberts, F. Couny, H. Sabert, B. Mangan, D. Williams, L. Farr, M. Mason, A. Tomlinson, T. Birks, J. Knight, and P. St. J. Russell, "Ultimate low loss of hollow-core photonic crystal fibres," Opt. Express, 13, pp. 236-244 (2005)

[8] J. Xu, C. Peucheret, "Two-Mode Multiplexing at 2x10.7Gbps over 7-cell hollow-core photonic band gap fiber," in ECOC 2011, paper We.10.P1.66

[9] J. K. Lyngs $\varnothing$, B. J. Mangan, C. Jakobsen, and P. J. Roberts, "7-cell core hollow-core photonic crystal fibers with low loss in the spectral region around $2 \mu \mathrm{m}$," Opt. Express 17, 23468 (2009).

[10] J. Carpenter, T. D. Wilkinson, "Precise modal excitation in multimode fibre for control of modal dispersion and mode-group division multiplexing," in ECOC 2011, paper We.10.P1.62

[11] T. G. Euser, G. Whyte, M. Scharrer, J. S. Y. Chen1, A. Abdolvand, J. Nold, C. F. Kaminski and P. St.J. Russell, "Dynamic control of higherorder modes in hollow-core photonic crystal fibers", Optics Express, 16, pp. 17972-17981 (2008)

[12] Pierre Sillard, Marianne Bigot-Astruc, David Boivin, Hélène Maerten, Lionel Provost, "Few-Mode Fiber for Uncoupled Mode-Division Multiplexing Transmissions," in ECOC 2011, paper Tu.5.LeCervin.7. 